\title{
Corpus
}

$10 \mid 2011$

Varia

\section{Les récits de vie comme corpus sociolinguistique : une approche discursive et interactionnelle}

Sandra Nossik

\section{OpenEdition}

1 Journals

Édition électronique

URL : http://journals.openedition.org/corpus/2045

DOI : $10.4000 /$ corpus.2045

ISSN : 1765-3126

Éditeur

Bases; corpus et langage - UMR 6039

Édition imprimée

Date de publication : 1 novembre 2011

Pagination : 119-135

ISSN : 1638-9808

\section{Référence électronique}

Sandra Nossik, «Les récits de vie comme corpus sociolinguistique : une approche discursive et interactionnelle », Corpus [En ligne], 10 | 2011, mis en ligne le 14 juin 2012, consulté le 07 septembre 2020. URL : http://journals.openedition.org/corpus/2045; DOI : https://doi.org/10.4000/corpus.2045 


\title{
Les récits de vie comme corpus sociolinguistique : une approche discursive et interactionnelle
}

\author{
Sandra NOSSIK \\ Université Paris Descartes / CEPED
}

Ce texte se propose de réfléchir sur la spécificité des récits de vie en tant que corpus de sociolinguistique, et, parallèlement, sur la spécificité de l'approche sociolinguistique des récits de vie.

Les entretiens biographiques sont depuis les années 1970 une méthode de recueil de données exploitée de diverses façons par la sociologie. Suivant ce modèle, les études sociolinguistiques ont également recours aux récits de vie, par exemple pour tenter de mettre au jour les représentations des locuteurs interrogés sur leurs pratiques linguistiques, ou bien pour éclairer des expériences de migration. L'utilisation d'un tel corpus en sociolinguistique diverge cependant de celle des sociologues par ses présupposés épistémologiques. Dans une première partie, nous exposerons ces différences de conception du corpus " récit de vie », qui tient au statut conféré à la parole des interviewés, et au rapport envisagé entre discours et réalité. Dans un second temps, sera abordée la nécessaire complémentarité entre une analyse discursive et une approche interactionnelle des récits de vie. Cette approche implique une conception "ouverte" du corpus, incluant le contexte situationnel et social de son recueil.

\section{Deux usages disciplinaires des récits de vie}

\subsection{Les récits de vie en sociologie}

Les entretiens biographiques sont depuis les années 1970 une méthode de recueil de données appréciée par la discipline sociologique. Dans la continuité des événements de mai 1968, et dans le cadre d'une remise en cause des grands modèles 


\section{S. NosSIK}

théoriques explicatifs tels que le structuralisme et le marxisme, la volonté de faire de la sociologie autrement se cristallisa en France dans l'approche biographique, dont le sociologue Daniel Bertaux est l'un des principaux représentants. En opposition aux systèmes idéologiques proposant des interprétations globales de la réalité sociale, ainsi qu'aux enquêtes quantitatives à la technicité croissante, le recours aux récits de vie se voulait une approche différente, visant à appréhender les faits sociaux à travers les dires de leurs acteurs. Avec une portée politique, elle se présentait également comme une occasion de rendre leur voix à des catégories de population privées de représentation dans les sphères institutionnelles ou académiques.

Cet intérêt renouvelé pour cette méthode dite qualitative fut dans le même temps impulsé par la redécouverte des travaux sociologiques de l'école de Chicago, notamment par l'ouvrage de William Isaac Thomas et Florian Znaniecki consacré aux migrants polonais aux Etats-Unis (The Polish Peasant in Europe and America, publié entre 1918 et 1920). Leur recherche se présente en effet sous la forme de cinq volumes dont le troisième est entièrement consacré à l'autobiographie de l'un de ces migrants, dont le récit de vie s'étend sur plus de trois cents pages. Ce récit, sollicité par les sociologues contre promesse de rémunération, semble avoir été rédigé par le témoin lui-même. Dans leur introduction au manuscrit, Thomas et Znaniecki soulignent « la supériorité des récits de vie sur tout autre type de matériau » sociologique, source la plus « complète » existante ([1919] $1998: 47)$.

Les entretiens biographiques devinrent ainsi une source de données fréquemment mobilisée, destinées à éclairer des questionnements sociologiques pouvant porter sur une classe sociale ou un métier spécifique («les ouvriers», "les enseignants »...), sur les expériences liées aux migrations, une classe d'âge, des questions de genre... (Heinritz \& Rammstedt 1991 : 340). Les interlocuteurs se voient ainsi choisis et regroupés par le chercheur suivant des catégories sociologiques préalables à l'enquête, caractérisant leur statut social ou leur parcours de vie, conformément aux orientations thématiques posées par lui au principe de la recherche. 
Les sociologues semblent aujourd'hui s'accorder sur la préférence de l'entretien dit «semi-directif» pour recueillir au mieux les témoignages des enquêtés. L'entretien semi-directif, ou «narratif» (Bertaux [1997] $2005: 11$ ), ou encore «interactif» (Bres 1999: 68), se veut une forme d'interaction proche de la conversation, grâce à l'adaptation continue des interrogations et interventions du chercheur à l'échange en cours. Il s'agit idéalement pour l'enquêteur de délimiter préalablement à la rencontre les thèmes à faire émerger durant l'entretien, puis d'adapter la forme et l'ordre de ses questions aux réactions de l'enquêté au fil de l'interaction. Pour ne pas réorienter les récits en cours, les « relances-miroir» (Demazière 2007: 90) reprenant les propos de l'interviewé sont préférées aux questions, qui pourraient lui imposer des catégorisations du monde préconçues par le chercheur. Au-delà de variations dans l'application de ces recommandations, c'est ce type d'entretien qui est devenu progressivement dans les sciences sociales « une norme académique enseignée comme une méthode canonique » (ibid.).

On ne saurait cependant parler d' «une» approche sociologique des récits de vie: la dénomination englobante d' « approche biographique » ou « qualitative » recouvre quantité de méthodes, variant autant dans les techniques de recueil de ces récits que dans les outils de leur analyse, et, plus profondément, dans les présupposés théoriques sous-tendant leur usage dans la recherche (Demazière 2008 : 15). Plus encore, il nous a paru que les textes consacrés réflexivement à l'utilisation des biographies en sociologie ne s'accordaient pas sur les termes mêmes de ces divergences. Nous tenterons donc à notre tour de rendre compte des diverses tendances théoriques décelables quant au rapport au type de corpus que sont les récits de vie dans la discipline sociologique. Nous verrons ainsi que l'hétérogénéité de ces approches nous semble pouvoir être comprise et problématisée à la lumière du lien conçu par les différents auteurs entre discours et réalité.

Dans le commentaire théorique précédant leur corpus, Thomas et Znaniecki notaient déjà que « tout devenir social est envisagé comme le produit d'une interaction continuelle entre conscience individuelle et réalité sociale objective» ([1919] 


\section{S. NossIK}

1998 : 45). Ce sont ainsi les rapports d'interdépendance reliant parcours individuels et structures sociales que la plupart des recherches sociologiques cherchent à mettre au jour par l'utilisation de récits de vie.

Dans un texte consacré aux diverses tendances à l'œuvre dans l'interprétation sociologique des biographies, Passeron distingue ainsi deux pôles théoriques autour desquels s'organisent selon lui les travaux contemporains. A la question de savoir «ce qui fait l'individuation d'une vie d'individu» (1990: 17), le premier cadre d'analyse, qu'il qualifie de « durkheimien », « subordonne l'intelligibilité biographique à la description des structures objectives [...] qui la précèdent et la déterminent» (ibid.) : le devenir individuel est, " avant toute possibilité de choix tactique ou stratégique, déjà structuré par des normes $[\ldots]$ socialement conditionnées [...] d'orientation biographique » (ibid. p. 18). Ce premier cadre théorique interprète donc les destins individuels comme conditionnés par un déterminisme social. Dans le cadre théorique contraire, baptisé "sartrien ", «l'action sociale des individus " influe cette fois au même titre que celle des structures sociales sur « le devenir biographique " (ibid. p. 17). En opposition à chacun de ces pôles, l'auteur pose pour sa part la nécessité de «saisir la structuration des biographies à la fois comme un effet des structurations longitudinales » déterminantes sous-jacentes «et comme le produit [...] que l'action sociale des individus inscrit, en aval, dans [...] ces structures longitudinales » (ibid. p. 20).

Nous retiendrons pour notre propos que les divergences dans l'exploitation sociologique des récits de vie semblent donc se résumer ici aux conceptions du déterminisme social soustendant leur analyse. Le fait que ces récits servent à mettre en relation trajectoires individuelles et structures sociales apparaît comme acquis : la fonction des récits de vie est de permettre un recueil d'informations sur le parcours social des enquêtés.

Pour Bertaux, les récits sont ainsi une source dont peuvent être extraites des données factuelles objectives sur l'objet analysé par le sociologue: "un entretien narratif [...] contient nécessairement bon nombre d'informations factuelles généralement exactes», permettant «d'étudier un fragment 
particulier de réalité sociale-historique, un objet social» ([1997] 2005: 12-14). Il distingue avec soin ces données factuelles des jugements subjectifs exprimés par les enquêtés, de «la subjectivité active» à l'œuvre dans l'entretien (1994: 223). Il s'agit par conséquent pour le sociologue de distinguer entre " données objectives" et "points de vue subjectifs », à l'aide de certains «moyens d'objectivation» (Beaud 1996 : 241): les dires des interviewés doivent être complétés et confrontés aux informations obtenues par d'autres méthodes d'enquête, telles que l'observation participante du chercheur. Stéphane Beaud et Florence Weber conseillent ainsi aux étudiants apprentis- sociologues : "adoptez toujours une position critique à l'égard des propos qu'on vous tient, recoupez toujours vos informations »; " vous chercherez à mettre en rapport les positions objectives des enquêtés, leurs pratiques telles que vous avez pu les observer et leurs points de vue subjectifs exprimés dans l'entretien » (1997: 266). Depuis une position qui semble plus savante que celle de l'enquêté, le sociologue exploite ainsi des données complémentaires à l'entretien pour fournir une analyse conforme à sa "perspective objectiviste » (Bertaux [1997] 2005 : 12).

Lorsque d'autres voies sont proposées pour une utilisation sociologique des entretiens biographiques, c'est sans remise en question de cette répartition constitutive entre faits objectifs et points de vue subjectifs. Ainsi, certaines recherches, qualifiées par Bertaux d'«herméneuticiennes» (1986: 27), s'intéressent quant à elles aux "points de vue indigènes " sur des expériences vécues (Demazière $2008: 16$ ), aux «attitudes » et « valeurs » des enquêtés (Thomas \& Znaniecki [1919] 1998 : 47), voire aux «conflits» entre «normes » sociales et désirs individuels (Schwartz 1990 : 177).

Si les objectifs de cette seconde approche sont différents, le sociologue s'intéressant aux opinions des enquêtés et non plus exclusivement à leurs parcours de vie, son présupposé épistémologique reste inchangé : les récits de vie offriraient à la fois des données factuelles objectives et des points de vue subjectifs, ces deux catégories d'informations pouvant être dissociées et exploitées séparément selon les souhaits scientifiques du chercheur. 


\section{S. NossIK}

Ce présupposé épistémologique à l'œuvre dans la plupart des travaux sociologiques implique une certaine conception du langage. Nous la qualifierons en suivant Lorenza Mondada de conception représentationnaliste du langage : le discours est un "véhicule neutre et transparent» transmettant sans obstacles un contenu informatif (2001 : 198). L'adéquation parfaite entre faits et mots permet un recueil de données sans équivoque par le chercheur: les informations «codées par le locuteur» sont "décodées symétriquement par son interlocuteur " (ibid.), dans une communication sans malentendu possible qui permet de considérer les récits de vie comme de simples comptes-rendus factuels de la pratique sociale.

Cette conception du lien entre langage et réalité suppose qu'il n'y a de subjectif que ce qui est explicité comme tel: seuls les jugements manifestement évaluatifs («c'était dur », "je n'ai pas aimé cette période») sont des données subjectives. C'est ce type d'énoncés que le sociologue est supposé repérer et distinguer des données factuelles offertes par le récit (Beaud 1996: 241). Une telle approche suppose en outre un chercheur à même de distinguer entre ce qui est vrai et ce qui relève de points de vue individuels.

\subsection{Les récits de vie en sociolinguistique}

Une autre approche possible consiste à envisager les récits de vie comme des «mises en intrigue». Dans le premier tome de sa trilogie intitulée Temps et Récit, Ricœur expose sa réflexion sur le concept aristotélicien de mimèsis, soit "l'imitation du réel », « la représentation du réel» dans les arts. Analysant plus particulièrement la forme narrative et le lien entre temps vécu et temps raconté, Ricœur insiste sur le caractère dynamique de la mimèsis : c'est un "processus actif », une « activité » de représentation, ou encore une «opération de configuration » (1983: 127). Cette "opération» procède à une transformation d'une suite d' événements» ponctuels en «une histoire» (ibid.), globale et synthétique : elle permet le passage d'une multiplicité de faits hétérogènes à une histoire cohérente. Loin d'être une restitution méticuleuse d'événements, un récit de vie est donc une « mise en intrigue » qui donne sens à ce qui est raconté, et en fait une histoire. Par le biais de l'activité narrative, les 
événements ponctuels et hétérogènes sont pris ensemble et configurés en une intrigue cohérente et signifiante.

Dans ce cadre, le matériau offert par les entretiens biographiques est précisément la reconstruction des réalités sociales dans les discours: c'est la re-configuration des histoires vécues qui en révèle la substance. Loin d'être des artefacts inutilisables dans un cadre scientifique parce que trop subjectifs ou narrativisés, les récits de vie constituent donc un corpus précieux en raison même de leur subjectivité, résultat d'une mise en mots et d'une mise en intrigue singulières.

De cette conception performative ou "praxéologique » (Mondada 2001: 198) du discours découlent deux conséquences méthodologiques : la première est la nécessité d'une analyse à partir de la matérialité discursive des récits (Pêcheux 1981). Puisque ce sont les dires des enquêtés qui permettent au chercheur d'accéder aux faits tels qu'ils sont re-produits, ces mises en mots, "traité[e]s comme des actions » (Mondada 2001 : 198), deviennent l'objet d'étude du chercheur. La seconde implication méthodologique de ce cadre théorique est la nécessaire prise en compte de la dimension interactionnelle de l'entretien: la construction sociale qu'est le récit doit être resituée dans «l'événement communicationnel» de l'entretien (ibid. p. 197), condition de son émergence.

\section{L'« entretien-texte » et l'« entretien-événement»}

\subsection{Une approche discursive}

A la conception représentationnaliste du discours, selon laquelle «il existerait une réalité neutre, indépendante de la perspective prise par l'observateur » (Gadet 2003 : en ligne), une approche sociolinguistique des récits de vie préférera donc une vision praxéologique du discours (Mondada 2001: 198), d'après laquelle «les dires recueillis construisent leur propre intelligibilité » (ibid., 197). Ce postulat implique une approche du sens des récits par l'étude de leur matérialité langagière : seule l'analyse formelle des choix discursifs des locuteurs permet d'éclairer le contenu de leurs récits.

Le corpus n'est plus appréhendé en tant que reflet du réel, mais comme une «construction interprétative du monde 


\section{S. NossIK}

social par certains de ses acteurs »(Blanchet 2007: 347). Le langage «typifie» les expériences humaines, «permettant de les ranger à l'intérieur de catégories élargies qui leur donnent un sens » (Berger \& Luckmann [1966] 1996: 58). C'est cette « constitution linguistique du monde» (Guilhaumou 2004: en ligne) qui est recherchée par l'analyse discursive des récits de vie.

Le caractère réflexif du langage permet une lecture herméneutique du corpus : dans les discours recueillis, «toute une série de catégories descriptives prennent la place du métadiscours ", offrant ainsi des ressources interprétatives internes au corpus (Guilhaumou 2004 : en ligne). Les descriptions et catégorisations linguistiques proposées par les locuteurs contiennent déjà en elles-mêmes réflexions et prises de positions. La tâche du chercheur est par conséquent " d'intégrer dans l'acte d'interprétation le langage des membres de la société étudiée, et le travail d'abstraction qui s'y déploie » (1998: 264). La description de la matérialité linguistique de son corpus permet ainsi d'accéder au "savoir social impliqué dans l'usage des formes linguistiques » (Guilhaumou et al. $1995: 65$ ).

Par exemple, l'étude des catégories descriptives épilinguistiques émergeant au fil des discours des enquêtés, telles que «langue » s'opposant à «dialecte» ou «bien parler» s'opposant à " mal parler », peut révéler l'appropriation (ou au contraire la mise à distance) de " discours antérieurs légitimant socialement ces catégories » (Canut 2007: 53). Notons également l'intérêt des tentatives discursives d'auto- catégorisation, soit de toutes les mises en mots ayant trait à la « construction de l'identité narrative du sujet parlant» (Deprez 2000: 168), qui peuvent mettre au jour hésitations et ruptures vis-à-vis des étiquettes officielles nationales, ethniques ou linguistiques (Nossik $2011: 8$ ).

En s'inscrivant dans la lignée du tournant interprétatif voire « herméneutique » de l'Analyse du Discours (Guilhaumou 2004 : en ligne), il s'agit donc de s'appuyer sur la « réflexivité des descriptions sociales » (2000: 116), c'est-à-dire sur le caractère à la fois pratique et cognitif des catégorisations linguistiques, pour analyser les récits de vie. 
Dans un ouvrage paru en 1997 et intitulé Analyser les entretiens biographiques, l'exemple de récits d'insertion, les sociologues Didier Demazière et Claude Dubar offrent une proposition exhaustive de méthode d'analyse des récits de vie, mise en application sur un corpus de récits d'insertion professionnelle de jeunes adultes. Bien que située dans un cadre disciplinaire sociologique, cette recherche prône et légitime une approche des entretiens par leur matérialité langagière, illustrant ce que peut être une analyse sociolinguistique de récits de vie.

La recherche des sociologues ne porte pas sur les informations factuelles contenues dans l'entretien, mais sur « la catégorisation sociale» mise en œuvre dans les récits, par laquelle « le sujet structure le sens de son monde social » (ibid., 37). Reposant sur le postulat que « les entretiens ne nous livrent jamais des "faits" mais des "mots" ( (ibid., 7), l'étude des sociologues ne s'occupe plus de classer des individus ou des trajectoires, ni des points de vue subjectifs ponctuels sur des faits objectifs, mais de mettre au jour « la forme symbolique et d'abord langagière - dans laquelle [les sujets] se racontent, argumentent et s'expliquent » (ibid., 304).

Pour analyser les récits et mettre au jour leurs « univers de croyance » (ibid., 335), Demazière et Dubar font le choix d'une méthode d'analyse de type structuraliste, inspirée principalement des travaux narratologiques de Barthes, et dans une moindre mesure de ceux de Propp et de Greimas. Sont donc recherchées « les oppositions et les corrélations les plus structurantes » (ibid., 7) des récits, leur signification étant susceptible d'être révélée par un «code du discours [...] fait de relations différentielles » (ibid., 95).

L'importance de l'interaction comme condition d'émergence du récit et composante de sa construction est posée théoriquement à de nombreuses reprises dans l'étude : les récits sont « une construction dialogique complexe » (1997: 7), et il faut «suivre le processus de l'activité signifiante dans l'interaction verbale et non verbale avec le chercheur » (ibid., 43). La dimension interactionnelle est cependant absente des analyses linguistiques des sociologues, qui se concentrent sur les réponses retranscrites des enquêtés. Comme le note Olivier 


\section{S. NosSIK}

Schwartz, «c'est une limite de la méthodologie proposée par Dubar et Demazière que de chercher exclusivement le sens d'un entretien du côté de sa mise en mots, en omettant de souligner qu'il réside aussi dans la voix qui porte le discours » (1999: 458). Les textes postérieurs de Demazière $(2007,2008)$ sont consacrés en revanche aux entretiens de recherche en tant qu'interactions.

\subsection{Une approche interactionnelle}

Si cette entrée dans le sens des discours par leur matérialité langagière marque donc une première rupture avec les analyses sociologiques positivistes des récits de vie, elle demeure cependant incomplète tant qu'elle n'est pas conjuguée avec une approche interactionnelle des récits : « recueillir de la parole par interview c'est recueillir une parole façonnée par l'interaction de l'interview » (Bres 1999: 69). Une approche sociolinguistique soucieuse des réalités du terrain ne saurait se contenter d'une analyse immanente de la dimension textuelle des récits. Parce que «l'entretien relève, comme toutes les pratiques langagières, de la catégorie de l'interaction verbale » (ibid., 62), il s'agit d'inclure dans le corpus son contexte de production, et d'appréhender dans toutes ses dimensions «l'événement communicationnel» (Mondada 2001 : 197) qu'est la conduite partagée d'un récit de vie. Les discours sont ainsi conçus comme « constitutivement lié[s] aux situations où il[s] apparai[ssent] » (ibid. p. 198), et l'analyse de «l'entretien-événement» est complémentaire de celle de «l'entretien-texte» (Schwartz 1999 : 458).

La prise en compte de l'entretien comme «événement communicationnel» et «format particulier d'interaction» (Mondada 2001 : 203) dans l'étude des récits ainsi recueillis implique une reconnaissance de l'enquêteur comme partie prenante de cette interaction (Bres 1999: 62, Gadet 2003: en ligne). A l'opposé du présupposé positiviste assimilant l'enquêteur aux biais nuisibles à l'authenticité des données recueillies, il s'agit d'accepter la présence du chercheur comme « une des composantes inévitables » de l'enquête, et de «l'incorporer de plein titre dans les analyses » (Mondada $1998: 59$ ). Le «paradoxe de l'observateur » théorisé par William Labov, c'est-à-dire « le problème consistant à observer comment les gens parlent 
quand ils ne sont pas observés ${ }^{1} \gg(1973$ : 82), incitait le sociolinguiste à élaborer diverses stratégies pour neutraliser sa présence durant l'enquête, en contournant par exemple par des procédés de diversion le cadre de l'interview initialement posé auprès des enquêtés ([1972] 1976 : 147). Cet idéal d'un observateur invisible n'a plus lieu d'être dans l'approche décrite ici, qui appréhende l'entretien de recherche comme une interaction au cours de laquelle « les interlocuteurs, y compris l'enquêteur, construisent collectivement une version du monde » (Mondada $2001:$ 197).

Les récits de vie sont ainsi des «produits collectifs 》 (Mondada 2001 : 199) : co-construits par les interactants en présence, ils s'élaborent autour des "relations que la personne construit sous nos yeux, avec nous et pour nous, entre ellemême et son univers social» (Deprez 1996: 166). De cette collaboration interactive spontanée, qui n'implique d'ailleurs aucunement une relation d'empathie ou de sympathie entre participants, il résultera des discours offrant une image de soi, un ethos, un "univers de croyance» (Demazière \& Dubar 1997 : 335), ou encore «des positionnements, des points de vue, des propositions contingentes sur le monde » (Mondada 2001 : 197). Bien qu'arrêtés sous forme textuelle après enregistrement et transcription, les récits n'en émanent pas moins de discours contingents, "qui se transforment séquentiellement au gré des ajustements entre interlocuteurs » (ibid., 214). Ainsi, si les récits de vie peuvent être appréhendés d'une part comme des productions textuelles finies, il apparaît d'autre part indispensable d'observer le processus de co-construction lui-même, l'élaboration interactive de contenus mouvants qui se déploie au fil de l'interaction (De Fina \& Perrino 2011).

La catégorisation de l'enquêteur comme incarnation d'une institution académique, d'une norme linguistique légitime, ou encore d'une instance de pouvoir symbolique, pourra ainsi entraîner un certain type de discours, qui éventuellement évolueront au fil de l'interaction et d'une re-catégorisation de

1 La traduction est de nous, l'extrait cité est le suivant: " the observer's paradox, the problem of observing how people speak when they are not being observed» (Labov 1973 : 82). 


\section{S. NossIK}

l'enquêteur. Les diverses marques linguistiques d'adresse sont par exemple des symptômes de ces « ajustements » (Demazière 2008), le passage du « vous » au « tu » n'étant pas rare au sein d'un entretien.

Après avoir insisté sur l'importance de la prise en compte de la co-construction des discours analysés, nous souhaiterions toutefois marquer une distance vis-à-vis de certains travaux sociolinguistiques se consacrant exclusivement à ces processus de négociation interactive du sens. Envisagés en termes de «co-construction », d' « ajustements réciproques », ou bien d' " offres de sens du chercheur », certains entretiens de recherche se voient décortiqués de façon exclusivement orientée sur cette collaboration, au point que le motif premier de la sollicitation de l'entretien de recherche auprès d'un informateur en est oublié. Que l'enquête porte initialement sur des parcours socio-professionnels, sur des expériences migratoires ou bien sur une pathologie psychiatrique, les discours recueillis ne se verront analysés qu'à la lumière de la coopération interactionnelle. Nous prenons pour notre part le parti de penser que les récits ont quelque chose à nous apprendre au-delà des "offres de sens du chercheur» en interaction : plutôt que comme une fin en soi, peut-être l'analyse de la co-construction des discours peut-elle être utilisée comme un des moyens d'accéder au contenu des récits de vie.

\subsection{Un corpus " ouvert"}

Une telle prise en compte du contexte situationnel de l'élaboration des récits de vie nécessite de s'interroger de nouveau sur ce que peut recouvrir le terme de « corpus ». La pertinence de la limite entre corpus et hors-corpus se voit discutée dans le cadre d'études ethnographiques, ces réflexions aboutissant à une « remise en cause de la confrontation entre le corpus et le horscorpus », comme ce fut le cas en Analyse du Discours française durant la décennie 1980 (Guilhaumou 2002 : en ligne).

Philippe Blanchet oppose ainsi les corpus de type « constitutif», sur lesquels se fonde exclusivement le travail du linguiste, aux corpus « restitutifs », qui n'offrent qu'un exemplier des données observables sur le terrain (2007 : 343). Dans le cadre d'une sociolinguistique ethnographique, il pose le statut 
« secondaire » $\mathrm{du}$ corpus vis-à-vis du terrain, dont «la complexité subjective des situations » ne saurait être reflétée par «un "corpus" même méthodiquement construit» (ibid.). Cette approche de l'objet de recherche implique un glissement des données analysées par le chercheur: les analyses ne sont plus fondées sur un corpus appréhendé en immanence, mais résultent au contraire de la familiarité du chercheur avec le terrain dans sa complexité, de sa «fréquentation réflexive et assidue » par observation participante (ibid.). C'est plus précisément un mouvement de va-et-vient entre le corpus proprement dit et les observations in situ qui en permet l'interprétation: "la fréquentation du terrain éclaire le "corpus" qui à son tour aide à rendre lisible la complexité du terrain » (ibid.). Le corpus se définit donc par sa "significativité » et non plus par sa « représentativité » (ibid., 347) : l'enjeu du corpus est son "efficacité signifiante» (ibid., 348) au regard des connaissances et des interprétations élaborées sur le terrain.

En opposition à l'analyse exclusivement textuelle de récits de vie proposée par Demazière et Dubar, Schwartz note également que «certaines ressources décisives pour l'interprétation se forment à travers la familiarité gagnée avec un "terrain" et l'espèce de connaissance "de l'intérieur" qu'elle apporte d'un univers de vie et de pensée » (1999: 460). Cette "connaissance de l'intérieur» pourrait être rapprochée du "savoir d'arrière-plan" (background knowledge) de Gumperz, cette connaissance des conventions socio-culturelles implicites (les «indices de contextualisation») permettant d'interpréter correctement ce qui est en jeu au cours d'une interaction ([1982] 1989: 64-65).

Les zones d'ombre des récits de vie, (pouvant avoir trait à des actions transgressant le cadre de la légalité, telles que les passages de frontières des migrants), ne peuvent par exemple être comprises que grâce à un savoir acquis sur le terrain, hors enregistrements. Il en va de même pour les références à des discours autres, plus ou moins explicites ou implicites, plus ou moins dévotes ou ironiques.

Sans abandonner la dimension textuelle du récit de vie proprement dit, il s'agit donc pour le sociolinguiste d'inclure 


\section{S. NossIK}

dans son corpus (sous forme de notes ethnographiques par exemple) le «savoir d'arrière-plan» acquis par l'observation participante, les échanges éphémères et les scènes informelles vécus durant l'enquête ethnographique. Construire un tel corpus " significatif» conduit ainsi à expliciter le rôle de ce savoir lors de l'exposition des analyses de la recherche, et non à le relativiser a posteriori en "idéalis[ant] une méthode au moins partiellement réinventée après-coup » (Blanchet 2009 : 149).

\section{Conclusion}

De par son interdisciplinarité constitutive, la sociolinguistique partage certaines méthodes d'enquête avec la sociologie, et dans le même temps s'en distingue par d'autres, suivant une spécificité épistémologique qui parfois semble perdre sa netteté. Il nous paraissait par conséquent nécessaire de nous efforcer de délimiter les ressemblances et les divergences d'avec cette discipline voisine mais non identique qu'est la sociologie, vis-à-vis du corpus commun que constituent les récits de vie.

Une approche sociologisante qui subordonne une parole subjective à des faits objectifs s'oppose en effet à une conception plus performative du discours, qui requiert une analyse des récits à partir de leur matérialité langagière. A l'aide d'une analyse discursive des récits de vie, il s'agit pour le sociolinguiste de mettre au jour des «façons de dire » le monde qui font témoignage. Les dires des narrateurs ne peuvent cependant être interprétés qu'à la lumière de la situation d'interaction dont ils sont issus. Une telle approche à la fois discursive et interactionnelle des récits de vie nécessite de consentir à un élargissement du corpus textuel, au profit d'observations et de connaissances issues du travail ethnographique du chercheur. Ainsi le sociolinguiste peut-il prétendre interpréter les reconstructions linguistiques du monde que sont les récits offerts par les narrateurs. 
Les récits de vie comme corpus sociolinguistique

\section{Références bibliographiques}

Beaud S. (1996). «L'usage de l'entretien en sciences sociales, Plaidoyer pour l' "entretien ethnographique" ", Politix 35 : 226-257.

Beaud S. \& Weber F. (1997). Guide de l'enquête de terrain, Produire et analyser des données ethnographiques. Paris : La Découverte.

Berger P. \& Luckmann T. (1966). The Social Construction of Reality. New York: Anchor Books. Trad. fr. (1996). La construction sociale de la réalité. Paris : Armand Colin.

Bertaux D. (1986). «Fonctions diverses des récits de vie dans le processus de recherche », in Desmarais D. \& Grell P. (éd.) Les récits de vie, Théorie, méthode et trajectoires types. Montréal : Saint Martin, 21-34.

Bertaux D. (1994). " Le modèle culturel des classes populaires russes face au passage à l'économie de marché », Revue d'Etudes Comparatives Est-Ouest 25 (4) : 197-228.

Bertaux D. ([1997] 2005). Le récit de vie. Paris : Armand Colin.

Blanchet P. (2007). «Sur le statut épistémologique de la notion de "corpus" dans un cadre ethno-sociolinguistique ", in Auzanneau M. \& Verdoïa S. (éd.) La mise en æuuvre des langues dans l'interaction. Paris : L'Harmattan, 341-352.

Blanchet P. (2009). «La réflexivité comme condition et comme objectif d'une recherche scientifique humaine et sociale », Cahiers de sociolinguistique 14 : 145-152.

Bres J. (1999). «L'entretien et ses techniques », in Calvet L.-J. \& Dumont P. (éd.) L'enquête sociolinguistique. Paris : L'Harmattan, 61-76.

Canut C. (2007). «L'épilinguistique en question », in Siouffi G. $\&$ Steuckardt A. (éd.) Les linguistes et la norme, Aspects normatifs du discours linguistique. Berne : Peter Lang, 4972.

De Fina A. \& Perrino S. (2011). "Interviews vs. "natural" contexts : A false dilemma », Language in Society $40: 1$ 11 . 


\section{S. NossiK}

Demazière D. (2007). «A qui peut-on se fier? Les sociologues et la parole des interviewés », Langage et société 121/122 : $85-100$.

Demazière D. (2008). «L'entretien biographique comme interaction. Négociations, contre-interprétations, ajustements de sens ", Langage et société $123: 15-35$.

Demazière D. \& Dubar C. (1997). Analyser les entretiens biographiques, l'exemple de récits d'insertion. Paris : Nathan.

Deprez C. (1996). «Parler de soi, parler de son bilinguisme, entretiens autobiographiques et récits de vie d'apprenants et de bilingues », AILE 7 : 155-180.

Deprez C. (2000). «Histoires de langues, histoires de vies », Cahiers de sociolinguistique 5 : 167-174.

Gadet F. (2003). « Derrière les problèmes méthodologiques du recueil des données », Texto! [en ligne], http://www. revue-texto.net/1996-2007/Inedits/Gadet_Principes.html

Guilhaumou J. (1998). L'avènement des porte-parole de la République (1789-1792). Essai de synthèse sur les langages de la Révolution française. Villeneuve-d'Ascq : Presses Universitaires du Septentrion.

Guilhaumou J. (2000). « De l'histoire des concepts à l'histoire linguistique des usages conceptuels », Genèses 38 : 105118.

Guilhaumou J. (2002). «Le corpus en analyse de discours: perspective historique », Corpus 1: [en ligne], http://corpus.revues.org/index $8 . h t m l$

Guilhaumou J. (2004). « Où va l'analyse de discours ? Autour de la notion de formation discursive », Texto! [en ligne], http://www.revue-texto.net/Inedits/Guilhaumou_AD.html

Guilhaumou J., Collinot A., Mazière F. \& Branca-Rosoff B. (1995). "Questions d'histoire et de sens ». Langages 29 (117) : 54-66.

Gumperz J. J. (1982). Discourse strategies. New York : Cambridge University Press. Trad. fr. (1989). Engager la conversation, Introduction à la sociolinguistique interactionnelle. Paris : Minuit. 
Heinritz C. \& Rammstedt A. (1991). «L'approche biographique en France », Cahiers internationaux de sociologie 91 : 330370.

Labov W. (1973). "The linguistic consequences of being a lame », Language in society $2: 81-115$.

Labov W. (1972). Sociolinguistic patterns. Philadelphia: University of Pennsylvania Press. Trad. fr. (1976). Sociolinguistique. Paris : Minuit.

Mondada L. (1998). "Technologies et interactions dans la fabrication du terrain du linguiste », Cahiers de l'ILSL 10 : 39-68.

Mondada L. (2001). "L'entretien comme événement interactionnel », in Grosjean M. \& Thibaud J.-P. (éd.) L'espace urbain en méthodes. Marseille : Parenthèses, 197-214.

Nossik S. (2011). «Dialogisme et positionnements politiques dans des récits de vie de migrants russophones ", in Actes du colloque Dialogisme: langues, discours. [en ligne], http://recherche.univ-montp3.fr/praxiling/spip.php?article2 64

Passeron J.-C. (1990). « Biographies, flux, itinéraires, trajectoires », Revue française de sociologie 31 (1) : 3-22.

Pêcheux M. (1981). "Ouverture", in Conein B. et al. (éd.) Matérialités discursives. Lille: Presses Universitaires de Lille, 15-18.

Ricœur P. (1983). Temps et Récit, Tome I, L'intrigue et le récit historique. Paris : Seuil.

Schwartz O. (1990). «Le baroque des biographies », Cahiers de philosophie $10: 173-183$.

Schwartz O. (1999). "Symposium sur Analyser les entretiens biographiques, L'exemple des récits d'insertion", Sociologie du travail 41 (4) : 453-461.

Thomas W. I. \& Znaniecki F. (1919). The Polish peasant in Europe and America, Monograph of an immigrant group, Volume 3 : Life record of an immigrant. Boston : Badger. Trad. fr. (1998). Le Paysan polonais en Europe et en Amérique, Récit de vie d'un migrant. Paris : Nathan. 